\title{
Air Kerma Rate Constants for Nuclides Important to Gamma Ray Dosimetry and Practical Application
}

\author{
Marko M. Ninkovic ${ }^{1}$ and Feriz Adrovic ${ }^{2}$ \\ 1 Institute of Nuclear Sciences - Vinca, Belgrade, \\ 2University of Tuzla, Faculty of Science, Tuzla, \\ 1 Serbia \\ ${ }^{2}$ Bosnia and Herzegovina
}

\section{Introduction}

It is often necessary to estimate the exposure rate at a distance from radionuclide emitting gamma or $\mathrm{X}$ rays. Such calculations may be required for planning radiation protection measures around radioactive sources, for calibration radiation monitoring instruments, for patient containing radionuclides or for estimating the absorbed dose to patients receiving brachytherapy. The factor relating activity and exposure rate has been various names: the $\mathrm{k}$ factor (Johns, 1961), the specific gamma ray constant (ICRU Rep. 10a, 1962), exposure rate constant (Parker et al., 1978) and gamma rate constant (Kereiakes \& Rosenstein, 1980). Conversion to SI units required that this factor be replaced by the air kerma rate constant $\Gamma_{\delta}$, which is now defined as:

$$
\Gamma_{\delta}=\frac{l^{2}}{A}\left(\frac{d K_{a i r}}{d t}\right)_{\delta}
$$

where $(\mathrm{dK} \text { air } / \mathrm{dt})_{\delta}$ is the air kerma rate due to photons of energy $>\delta$, at a distance $l$ from a point source of activity $A$. The SI unit for $\Gamma_{\delta}$ is $\mathrm{J} \mathrm{m}^{2} \mathrm{~kg}^{-1}$ which, when the terms gray and becquirel are used, becoms Gy $\mathrm{m}^{2} \mathrm{~s}^{-1} \mathrm{~Bq}^{-1}$.

In the process of analysing accessible data on the are kerma rate constants and its precursos for many radionuclides often used in practice (Nachtigal, 1969; Ninkovic \& Mladenovic, 1970; NCRP Rep. 49, 1976; Ungar \& Trabey, 1982; Aird et al., 1984; Attix, 1986; Ninkovic, 1987; Wasserman \& Groenwald, 1988; Ninkovic \& Raicevic, 1992,1993; Sabol \& Weng, 1995; Ninkovic et al., 2005) it was concluded that published data are in strong disagreement. That is the reason we decided to recalculate this quantities on the basis of the latest data on gamma ray spectra and on the latest data for mass energy-transfer coefficients for air.

\section{Derivation of the equation for calculation of $\Gamma_{\delta}$}

The kerma $\mathrm{K}_{\mathrm{air}}$, for interaction of $\mathrm{X}$-rays and gamma rays with air is given by: 


$$
K_{\mathrm{air}}=\Phi \frac{\mu_{k}}{\rho} E
$$

where $\Phi$ is the flunce, $E$ the photon energy, and $\mu_{\kappa} / \rho$ the energy-dependent mass energytransfer coefficient for air.

The kerma rate, $\mathrm{d} K / \mathrm{d} t$, is obtained from the kerma by substituting the flux density $\psi$ for the fluence $\Phi$ in Equation 2:

$$
\frac{d K_{a i r}}{d t}=\psi \frac{\mu_{k}}{\rho} E
$$

where $\psi$ is expressed in $\mathrm{m}^{-2} \mathrm{~s}^{-1}$. The quantity $\psi$ is derived from the activity $A$, of a radiation source in accordance with inverse square low:

$$
\psi=\frac{A}{4 \pi l^{2}}
$$

By inserting Equation 4 in Equation 3, the following equation is obtained:

$$
\frac{d K_{a i r}}{d t}=\frac{A}{4 \pi l^{2}} \frac{\mu_{k}}{\rho} E
$$

If photons with energy $E_{i}$ are emitted per decay event with yield $p_{i}$, Equation 5 becomes:

$$
\frac{d K_{\text {air }}}{d t}=\frac{A}{4 \pi l^{2}} \sum_{i}\left(\frac{\mu_{k}}{\rho}\right)_{i} p_{i} E_{i}
$$

By inserting Equation 6 in Equation 1, the following equation is obtained for $\Gamma_{\delta}$ :

$$
\Gamma_{\delta}=\frac{1}{4 \pi} \sum_{i}\left(\frac{\mu_{k}}{\rho}\right)_{i} p_{i} E_{i}
$$

\section{Calculation of $\Gamma_{\delta}$}

Starting from Equation 7, the air kerma rate constants, $\Gamma_{\delta}$, were calculated using data on mass energy-transfer coefficients for air (Hubbell, 1969; Hubbell \& Seltzer, 2001) and data on photon emission yield in the process of decay of the radionuclides (Firestone, 1996; Stabin \& Luz, 2002). The subscript $\delta$, implies that only photons with energy $>\delta$, in $\mathrm{MeV}$ are included in the calculation.

Concerning the radiation spectra emitted per decay of a radionuclide, there are three types of photons: the gamma ray photons, those characteristic X-ray photons, those from internal conversion of gamma rays and electron capture and those accompanying bremsstrahlung processes of electrons from $\beta^{-}$decay and internal conversion of gamma rays and $X$ rays. In this calculation gamma rays and characteristic $X$-ray photons with energies $>20 \mathrm{keV}$ as $\delta$ - value are only ones to have been taken into account. The contribution of bremsstrahlung radiation has not been included. 
In the calculation, instead of gamma ray total transition intensities, the gamma ray intensities corrected for internal conversion of gamma rays were used.

The particular air kerma rate constants were calculated for each discrete line of the photon spectrum of the radionuclide, with effective yield per decay $>0.01 \%$ and energy $>20 \mathrm{keV}$. Since the energy structure of the photon spectra and accessible discrete numerical values of the mass energy-transfer coefficient for air are not the same, the cubic spline interpolation was used to calculate the coefficient, where photon spectrum data are available.

\section{Results}

\subsection{New recalculated values of $\Gamma_{\delta}$}

Table 1, lists recalculated air kerma rate constants for the 35 radionuclide used most often in gamma ray dosimetry and practical applications. For every radionuclide in the table are given the following data:

in column 1 the symbol of gamma-emitting nuclide,

in column 2 the half-life,

in column 3 the low- energy photon spectra limit,

in column 4 the high-energy photon spectra limit,

in column 5 the calculated value of the constant in basic SI units, and finaly

in column 6 the calculated value of the constant in practical units $\left(\mu \mathrm{Gy} \mathrm{m} \mathrm{m}^{2} \mathrm{GBq}^{-1} \mathrm{~h}^{-1}\right)$

The last unit, for air kerma rate constant, is the practical one especially, for radiation protection and safety calculations in nuclear medicine laboratories, industrial radiography and many others applications of point gamma radiation sources.

The accuracy of calculation of air kerma rate constants is not more than three significant figures. The major portion of the standard error associated with these calculated values of $\Gamma_{\delta}$ arise from uncertainties in relative intensity measurements of the $\mathrm{X}$ ray and gamma ray photon spectra and intensity of omitted bremsstrahlung radiation.

Bremsstrahlung radiation contributes to the total air kerma rate constant by, for exam $\leq \leq \mathrm{le}$, for ${ }^{60} \mathrm{Co}$, not more than $0.4 \%$, and this decreases markedly with decreasing photon energy (BCRUM, Br.J.Rad., 55, 1982). The contribution to $\Gamma_{\delta}$ from the omitted photons of energies < $20 \mathrm{keV}$, varies from radionuclide to radionuclide, this is not interesting for the purposes of practical health physics, but is of interest in specific nuclear medicine radionuclide applications.

\subsection{Examples of our previous measurements of photon spectra and calculation of $\Gamma_{\delta}$ for selected radionuclide}

The next section of the text shows, as examle, the data of our previous measurement of the photon spectrum and the results of calculating the air kerma rate constants for the three selected radionuclides ( ${ }^{182} \mathrm{Ta}$, ${ }^{192} \mathrm{Ir}$ and ${ }^{226} \mathrm{Ra}$ in equilibrium with its decay products).

\subsubsection{Photon spectra and recalculated of $\Gamma_{\delta}$ for ${ }^{182}$ Ta radionuclide}

As can be seen from Table 2, the entire of photon ray spectrum of ${ }^{182} \mathrm{Ta}$ is divided into five characteristic groups of photon lines. The air kerma rate constant was calculated for every 


\begin{tabular}{|c|c|c|c|c|c|}
\hline \multirow[t]{2}{*}{ Radionuclide } & \multirow[t]{2}{*}{ Half - life } & \multicolumn{2}{|c|}{ Energy interval (MeV) } & \multicolumn{2}{|c|}{ Air kerma rate constant } \\
\hline & & from & to & aGy $\mathrm{m}^{-2} \mathrm{~Bq}^{-1} \mathrm{~s}^{-1}$ & $\mu \mathrm{Gy} \mathrm{m} \mathrm{m}^{-2} \mathrm{GBq}^{-1} \mathrm{~h}^{-1}$ \\
\hline${ }^{11} \mathrm{C}$ & $20.38 \mathrm{~min}$ & - & 0.5110 & 38.7 & 139.3 \\
\hline${ }^{13} \mathrm{~N}$ & $9.965 \mathrm{~min}$ & - & 0.5110 & 38.7 & 139.4 \\
\hline $15 \mathrm{O}$ & $2.037 \mathrm{~min}$ & - & 0.5110 & 38.7 & 139.5 \\
\hline $18 \mathrm{~F}$ & $109.8 \mathrm{~min}$ & 0.0005 & 0.5110 & 37.5 & 135.1 \\
\hline${ }^{24} \mathrm{Na}$ & $14.96 \mathrm{~h}$ & 1.3690 & 3.8660 & 121.3 & 436.7 \\
\hline $42 \mathrm{~K}$ & $12.36 \mathrm{~h}$ & 0.3126 & 2.4240 & 9.10 & 32.8 \\
\hline $43 \mathrm{~K}$ & $22.3 \mathrm{~h}$ & 0.2206 & 1.3940 & 35.5 & 127.8 \\
\hline${ }^{51} \mathrm{Cr}$ & $27.70 \mathrm{~h}$ & 0.0005 & 0.3201 & 1.17 & 4.22 \\
\hline${ }^{52} \mathrm{Fe}$ & $8.275 \mathrm{~h}$ & 0.0006 & 1.0399 & 27.01 & 97.24 \\
\hline${ }^{59} \mathrm{Fe}$ & $44.50 \mathrm{~d}$ & 0.0069 & 1.4817 & 40.54 & 145.9 \\
\hline $57 \mathrm{Co}$ & $271.74 \mathrm{~d}$ & 0.0007 & 0.6924 & 3.92 & 14.11 \\
\hline${ }^{58} \mathrm{Co}$ & $70.86 \mathrm{~d}$ & 0.0007 & 1.6747 & 35.84 & 129.0 \\
\hline${ }^{60} \mathrm{Co}$ & $5.271 \mathrm{a}$ & 1.1732 & 1.3325 & 85.82 & 309.0 \\
\hline${ }^{67} \mathrm{Ga}$ & $3.261 \mathrm{~d}$ & 0.0010 & 0.8877 & 5.40 & 19.45 \\
\hline${ }^{68} \mathrm{Ga}$ & $1.127 \mathrm{~h}$ & 0.0010 & 1.8830 & 35.84 & 129.0 \\
\hline $75 \mathrm{Se}$ & $119.79 \mathrm{~d}$ & 0.0013 & 0.5722 & 13.40 & 48.25 \\
\hline${ }^{99} \mathrm{Mo}$ & $65.94 \mathrm{~h}$ & 0.0024 & 0.9608 & 5.49 & 19.77 \\
\hline $99 \mathrm{mTc}$ & $6.01 \mathrm{~h}$ & 0.0024 & 0.1426 & 3.92 & 14.10 \\
\hline 111In & $67.31 \mathrm{~h}$ & 0.0031 & 0.2454 & 23.09 & 83.13 \\
\hline $113 \mathrm{mIn}$ & $99.49 \mathrm{~min}$ & 0.0033 & 0.3917 & 12.22 & 44.00 \\
\hline $123 I$ & $13.27 \mathrm{~h}$ & 0.0038 & 0.7836 & 10.0 & 36.1 \\
\hline $125 \mathrm{I}$ & $59.4 \mathrm{~d}$ & 0.0038 & 0.0355 & 10.48 & 37.73 \\
\hline 131I & $8.021 \mathrm{~d}$ & 0.0041 & 0.7229 & 14.50 & 52.20 \\
\hline${ }^{127} \mathrm{Xe}$ & $36.4 \mathrm{~d}$ & 0.0039 & 0.6184 & 14.19 & 51.09 \\
\hline${ }^{133} \mathrm{Xe}$ & $5.243 \mathrm{~d}$ & 0.0043 & 0.1606 & 3.98 & 14.33 \\
\hline${ }^{137} \mathrm{Cs} /{ }^{137} \mathrm{Ba}$ & $30.04 \mathrm{a}$ & 0.0045 & 0.6617 & 22.80 & 82.10 \\
\hline 152 $\mathrm{Eu}$ & $13.537 \mathrm{a}$ & 0.0056 & 1.7691 & 41.36 & 148.9 \\
\hline${ }^{154} \mathrm{Eu}$ & $8.593 \mathrm{a}$ & 0.0061 & 1.5965 & 44.23 & 159.2 \\
\hline${ }^{170} \mathrm{Tm}$ & $128.6 \mathrm{~d}$ & 0.0070 & 0.0843 & 0.154 & 0.554 \\
\hline${ }^{182 \mathrm{Ta}}$ & $114.43 \mathrm{~d}$ & 0.0084 & 1.4531 & 44.45 & 160.0 \\
\hline 192Ir & $73.827 \mathrm{~d}$ & 0.0089 & 1.0615 & 30.30 & 109.1 \\
\hline $197 \mathrm{Hg}$ & $2.672 \mathrm{~d}$ & 0.0097 & 0.2687 & 3.159 & 11.37 \\
\hline${ }^{198} \mathrm{Au}$ & $2.695 \mathrm{~d}$ & 0.0100 & 1.0877 & 15.15 & 54.54 \\
\hline${ }^{201} \mathrm{Tl}$ & $3.038 \mathrm{~d}$ & 0.0058 & 0.1674 & 2.84 & 10.22 \\
\hline${ }^{241} \mathrm{Am}$ & $432.2 \mathrm{a}$ & 0.0139 & 0.1030 & 1.102 & 3.97 \\
\hline
\end{tabular}

Table 1. Air kerma rate constant for some radionuclide considering photon energy above $20 \mathrm{keV}$

discrete photon line with yield per decay event $>0.01 \%$ and starting with energy of 0.03174 $\mathrm{MeV}$ as the delta value. That means that four characteristic X-ray lines are included. The group and total air kerma rate constant are obtained then by addition of partiale or single photon lines constant. Finally, a value of $(44.8 \pm 0.9)$ aGy m${ }^{2} \mathrm{~s}^{-1} \mathrm{~Bq}^{-1}$ for an unshielded ${ }^{182} \mathrm{Ta}$ source has been obtained. That value is in good agreement with a new recalculated value given in Table 1. 
Bearing in mind that standard tantalum sources are usually packed into $0.1 \mathrm{~mm}$ of platinum, it was calculated the constant for this type of source also. For that goal, it was calculated the absorption of tantalum photons into $0.1 \mathrm{~mm}$ of platinum and obtained that in this way the air kerma rate constant is reduced by $4,46 \%$. After this correction, a value of (42.8 \pm 0.9$)$ aGy m$~_{2} \mathrm{~s}^{-1} \mathrm{~Bq}^{-1}$ was obtained for air kerma rate constant for standard packaged encapsulated tantalum source (Ninkovic \& Raicevic, 1992).

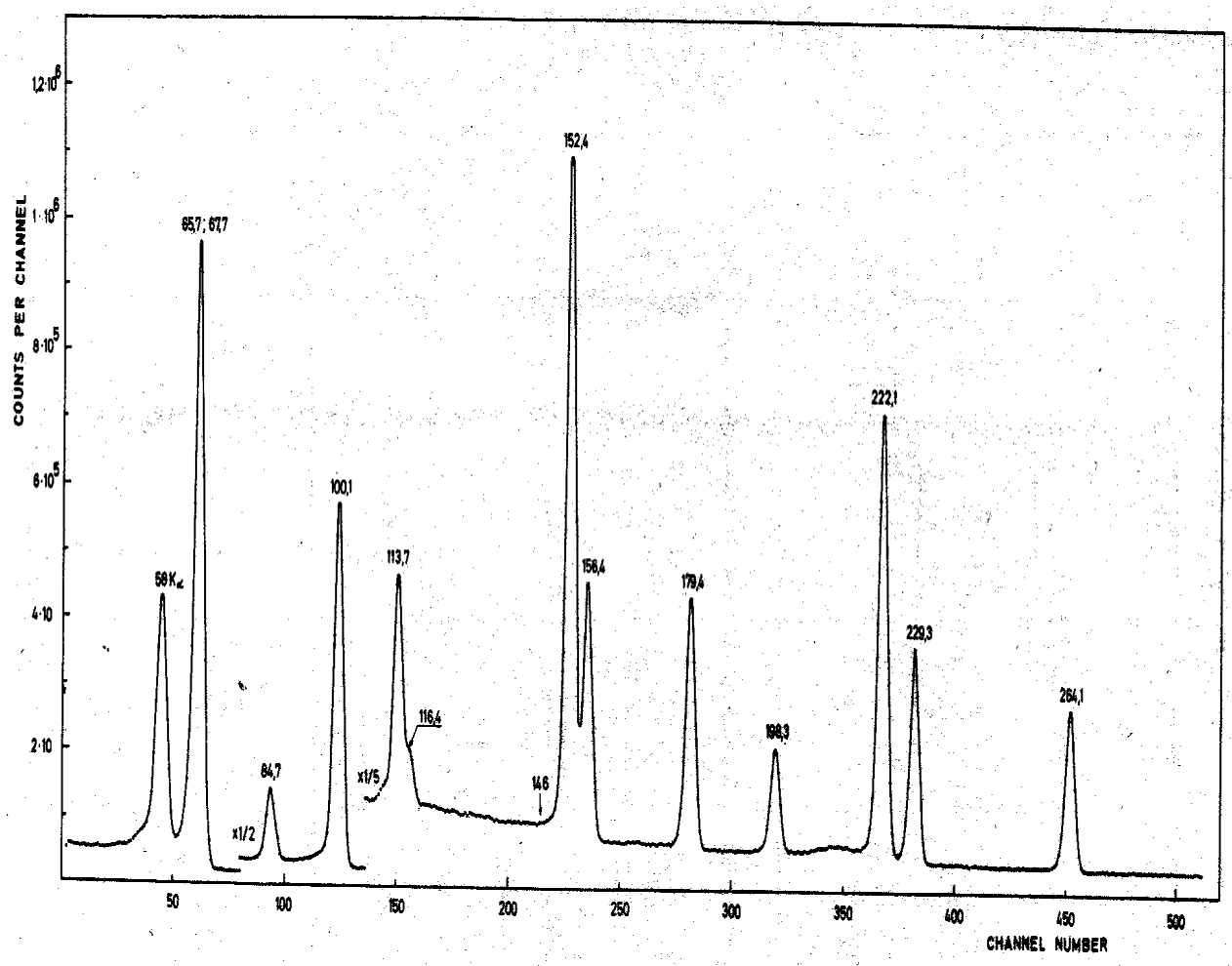

Fig. 1a. Low energy region $(50-300 \mathrm{keV})$ of the photons spectrum emitted in decay of ${ }^{182} \mathrm{Ta}$ radionuclide 




Fig. 1b. Middle energy region $(250-1200 \mathrm{keV})$ of the photons spectrum emitted in decay of ${ }^{182} \mathrm{Ta}$ radionuclide

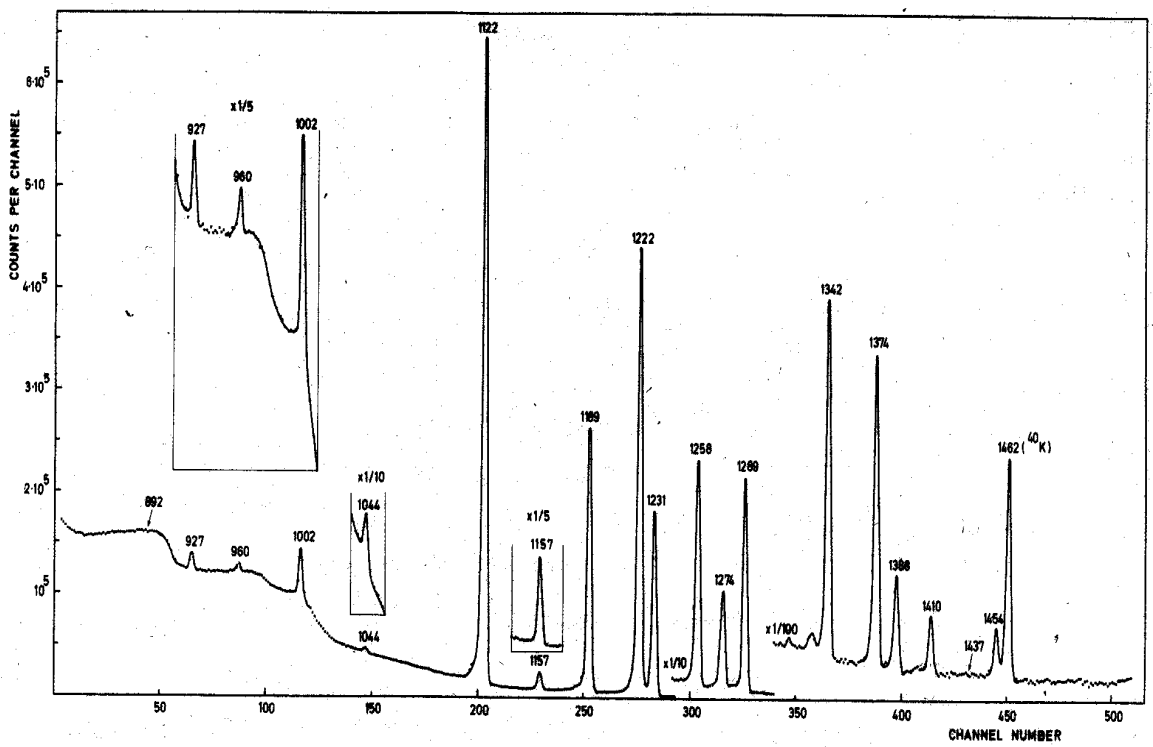

Fig. 1c. High energy region $(800-1500 \mathrm{keV})$ of the photons spectrum emitted in decay of ${ }^{182} \mathrm{Ta}$ radionuclide 


\begin{tabular}{|c|c|c|c|c|c|}
\hline Group of lines & $\begin{array}{l}\text { Energy } \\
{[\mathrm{MeV}]}\end{array}$ & $\begin{array}{l}\text { Yield per } \\
\text { decay } \\
{[\%]}\end{array}$ & $\begin{array}{l}\text { Air mass energy } \\
\text { transfer coeff. } \\
{\left[10^{-3} \mathrm{~m}^{2} \mathrm{~kg}^{-1}\right]}\end{array}$ & 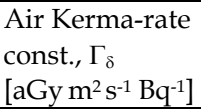 & $\begin{array}{l}\text { Yield to } \\
\text { total } \Gamma_{\delta} \\
{[\%]}\end{array}$ \\
\hline 1 & 2 & 3 & 4 & 5 & 6 \\
\hline \multirow[t]{9}{*}{$\mathrm{I}$} & 0.03174 & $0.46 \pm 0.07$ & 12.780 & $0.02 \pm 0.003$ & 0.05 \\
\hline & 0.04272 & $0.24 \pm 0.02$ & 5.650 & $0.01 \pm 0.001$ & 0.02 \\
\hline & $0.05798^{(*)}$ & $10.1 \pm 0.4$ & 3.145 & $0.24 \pm 0.01$ & 0.53 \\
\hline & $0.05932^{(*)}$ & $17.6 \pm 0.6$ & 3.045 & $0.41 \pm 0.02$ & 0.91 \\
\hline & 0.06572 & $3.00 \pm 0.07$ & 2.720 & $0.07 \pm 0.002$ & 0.16 \\
\hline & $0.06695^{(*)}$ & $2.1 \pm 0.1$ & 2.760 & $0.05 \pm 0.002$ & 0.11 \\
\hline & $0.0672^{(*)}$ & $7.5 \pm 0.3$ & 2.665 & $0.17 \pm 0.01$ & 0.38 \\
\hline & 0.06775 & $41.0 \pm 1.0$ & 1.645 & $0.94 \pm 0.02$ & 2.10 \\
\hline & \multicolumn{4}{|l|}{$1.91 \pm 0.1$} & 4.26 \\
\hline \multirow[t]{12}{*}{ II } & 0.08468 & $2.6 \pm 0.3$ & 2.352 & $0.07 \pm 0.01$ & 0.16 \\
\hline & 0.10011 & $14.0 \pm 0.4$ & 2.320 & $0.41 \pm 0.01$ & 0.92 \\
\hline & 0.11367 & $1.90 \pm 0.04$ & 2.345 & $0.06 \pm 0.01$ & 0.13 \\
\hline & 0.11642 & $0.43 \pm 0.01$ & 2.350 & $0.02 \pm 0.001$ & 0.04 \\
\hline & 0.15243 & $6.9 \pm 0.1$ & 2.500 & $0.34 \pm 0.01$ & 0.76 \\
\hline & 0.15639 & $2.7 \pm 0.2$ & 2.520 & $0.14 \pm 0.01$ & 0.31 \\
\hline & 0.17639 & $3.1 \pm 0.2$ & 2.595 & $0.18 \pm 0.01$ & 0.40 \\
\hline & 0.19835 & $1.5 \pm 0.1$ & 2.665 & $0.10 \pm 0.01$ & 0.22 \\
\hline & 0.22211 & $7.4 \pm 0.2$ & 2.735 & $0.57 \pm 0.12$ & 1.27 \\
\hline & 0.22932 & $3.7 \pm 0.1$ & 2.750 & $0.30 \pm 0.01$ & 0.67 \\
\hline & 0.26408 & $3.6 \pm 0.1$ & 2.830 & $0.34 \pm 0.01$ & 0.76 \\
\hline & \multicolumn{4}{|c|}{$2.53 \pm 0.08$} & 5.64 \\
\hline \multirow[t]{5}{*}{ III } & 0.92798 & $0.63 \pm 0.02$ & 2.830 & $0.21 \pm 0.01$ & 0.47 \\
\hline & 0.95872 & $0.36 \pm 0.05$ & 2.815 & $0.12 \pm 0.02$ & 0.27 \\
\hline & 1.00170 & $2.1 \pm 0.1$ & 2.810 & $0.75 \pm 0.04$ & 1.67 \\
\hline & 1.04443 & $0.24 \pm 0.01$ & 2.775 & $0.09 \pm 0.004$ & 0.20 \\
\hline & \multicolumn{4}{|c|}{$1.17 \pm 0.22$} & 2.61 \\
\hline \multirow[t]{10}{*}{ IV } & 1.11341 & $0.38 \pm 0.07$ & 2.752 & $0.15 \pm 0.03$ & 0.33 \\
\hline & 1.12130 & $35.0 \pm 0.7$ & 2.750 & $13.76 \pm 0.27$ & 30.71 \\
\hline & 1.1575 & $0.98 \pm 0.06$ & 2.730 & $0.39 \pm 0.02$ & 0.87 \\
\hline & 1.18905 & $16.3 \pm 0.3$ & 2.710 & $6.70 \pm 0.12$ & 14.96 \\
\hline & 1.22141 & $27.2 \pm 0.5$ & 2.700 & $11.46 \pm 0.21$ & 25.58 \\
\hline & 1.23102 & $11.6 \pm 0.4$ & 2.750 & $4.92 \pm 0.17$ & 10.98 \\
\hline & 1.25742 & $1.50 \pm 0.05$ & 2.690 & $0.65 \pm 0.03$ & 1.45 \\
\hline & 1.27373 & $0.65 \pm 0.01$ & 2.670 & $0.28 \pm 0.01$ & 0.63 \\
\hline & 1.28916 & $1.35 \pm 0.03$ & 2.665 & $0.59 \pm 0.02$ & 1.32 \\
\hline & \multicolumn{4}{|c|}{$38.90 \pm 0.82$} & 86.83 \\
\hline \multirow[t]{6}{*}{ V } & 1.34273 & $0.27 \pm 0.01$ & 2.645 & $0.12 \pm 0.01$ & 0.27 \\
\hline & 1.37384 & $0.22 \pm 0.01$ & 2.635 & $0.10 \pm 0.01$ & 0.22 \\
\hline & 1.38740 & $0.09 \pm 0.01$ & 2.625 & $0.04 \pm 0.01$ & 0.09 \\
\hline & 1.41010 & $0.05 \pm 0.01$ & 2.618 & $0.02 \pm 0.01$ & 0.04 \\
\hline & 1.45305 & $0.04 \pm 0.01$ & 2.600 & $0.02 \pm 0.01$ & 0.04 \\
\hline & \multicolumn{4}{|c|}{$0.30 \pm 0.003$} & 0.66 \\
\hline \multicolumn{5}{|c|}{ Total air-kerma rate constant $44.8 \pm 0.9$} & 100.0 \\
\hline
\end{tabular}

${ }^{(*)}$ - Characteristic X-ray $\mathrm{K} \alpha_{2}, \mathrm{~K} \alpha_{1}, \mathrm{~K} \beta \square$ and $\mathrm{K} \beta_{1}$ respectively

Table 2. Data for calculation and calculated Partial, Groups and Total Air kerma rate constant of ${ }^{182}$ Ta radionuclide (Ninkovic \& Raicevic, 1992) 


\subsubsection{Photon spectra and calculated of $\Gamma_{\delta}$ for ${ }^{192} \mathrm{Ir}$ radionuclide}

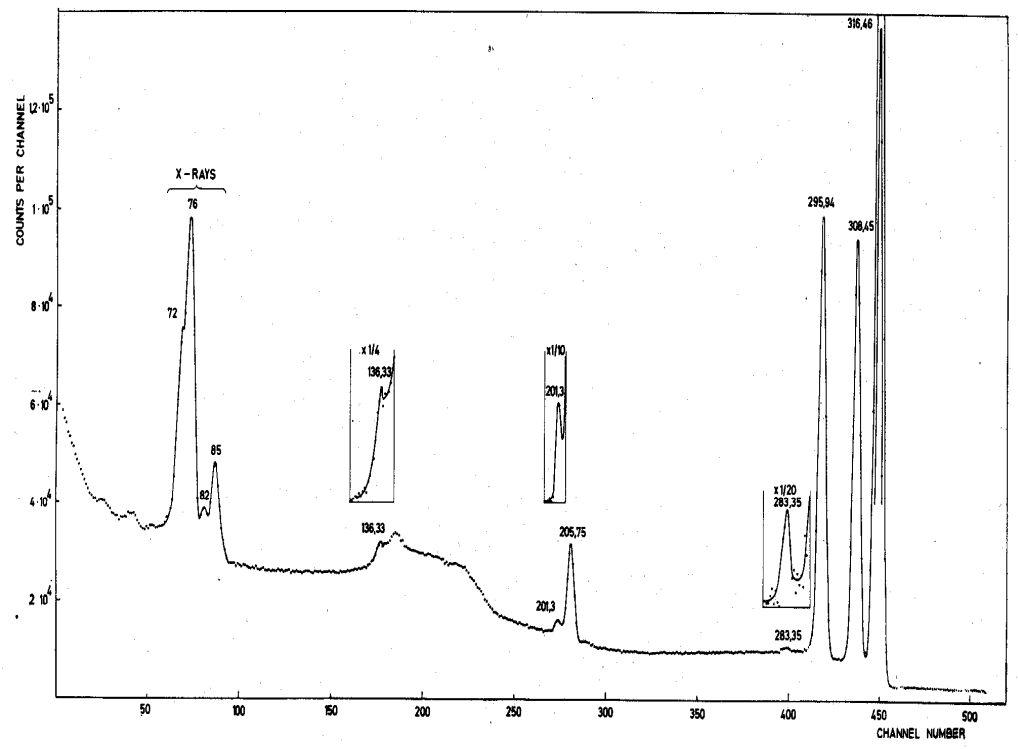

Fig. 2a. Energy spectrum of the photons emitted in decay of ${ }^{192}$ Ir radionuclide in energy interval from 50 to $350 \mathrm{keV}$

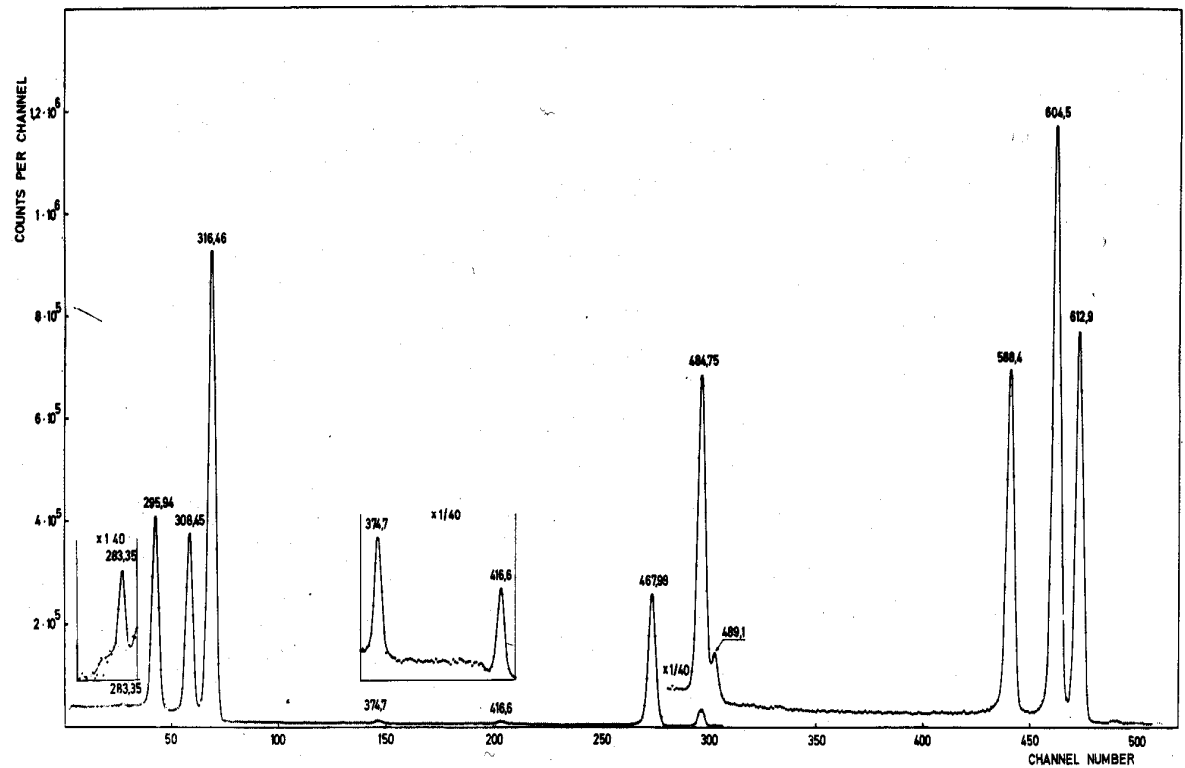

Fig. 2b. Energy spectrum of the photons emitted in decay of ${ }^{192}$ Ir radionuclide in energy interval from 250 to $650 \mathrm{keV}$ 


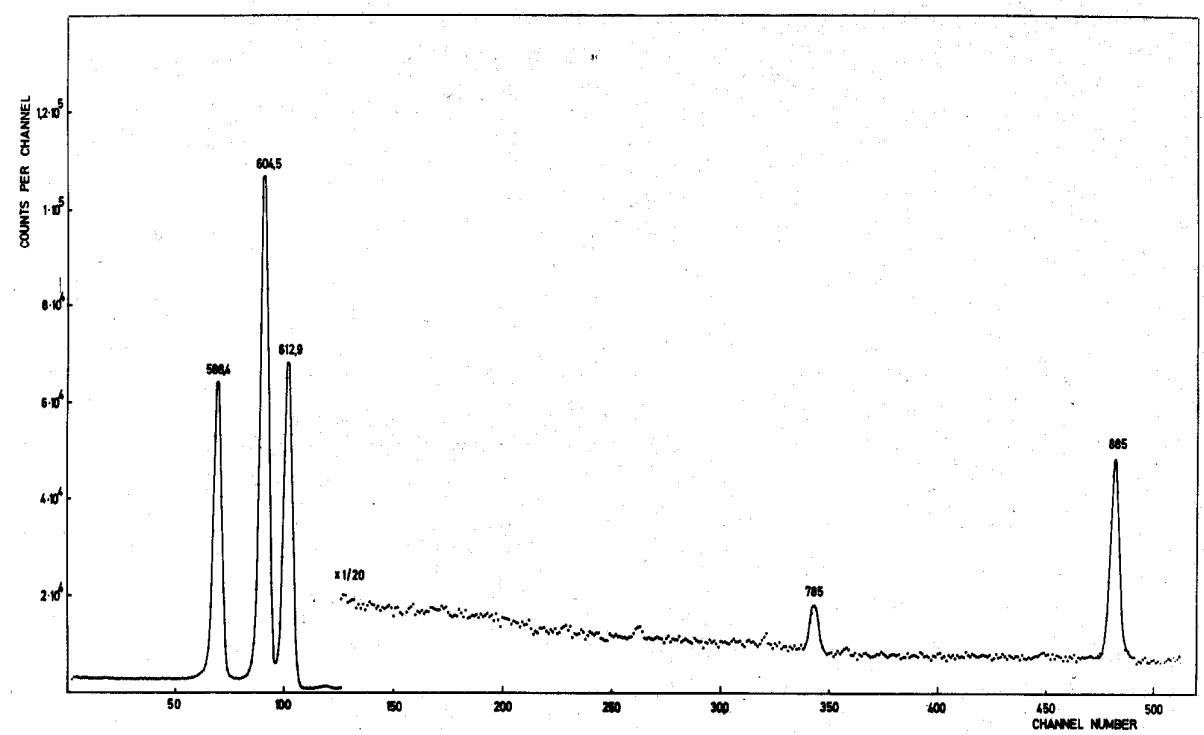

Fig. 2c. Energy spectrum of the photons emitted in decay of ${ }^{192}$ Ir radionuclide in energy interval from 550 to $900 \mathrm{keV}$

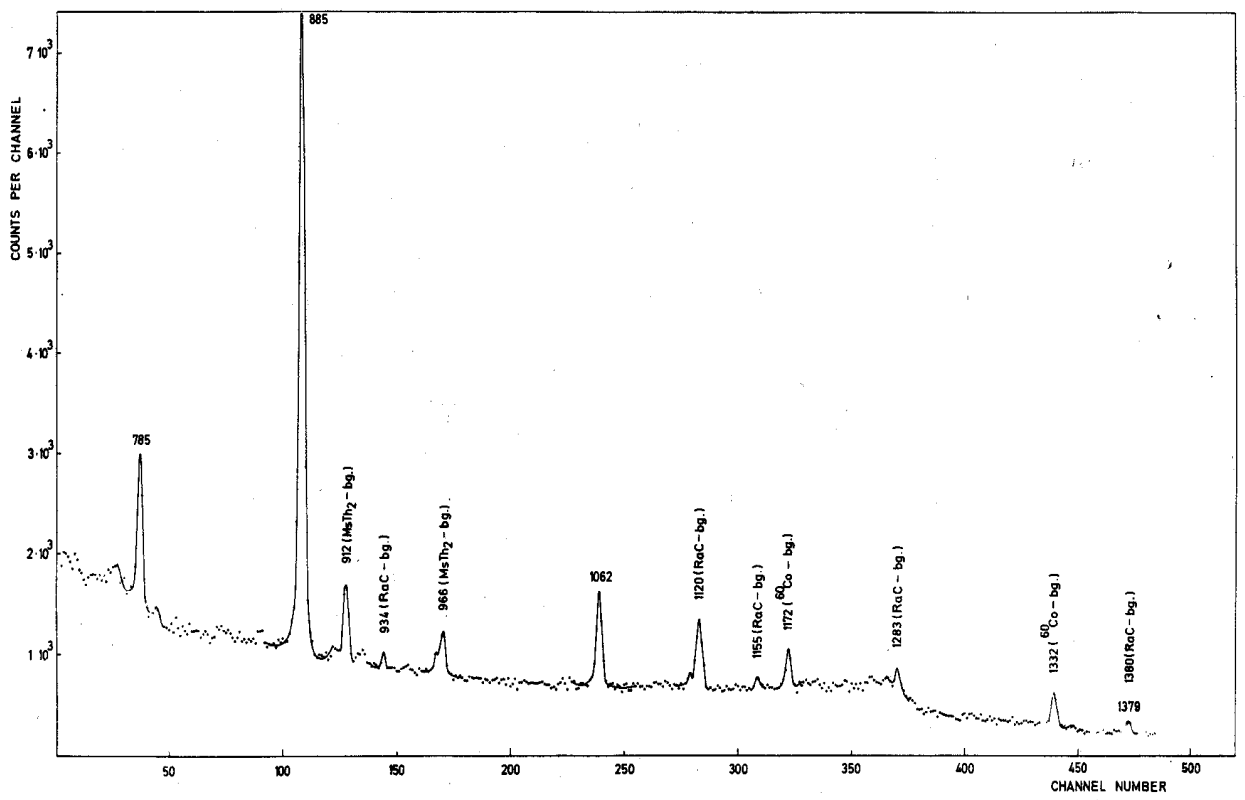

Fig. 2d. Energy spectrum of the photons emitted in decay of ${ }^{192}$ Ir radionuclide in energy interval from 750 t0 $1400 \mathrm{keV}$. This part of the spectrum contains many background lines from the $\mathrm{RaC}$, MsTh and ${ }^{60} \mathrm{Co}$ 


\begin{tabular}{|c|c|c|c|c|c|}
\hline $\begin{array}{l}\text { Group of } \\
\text { lines }\end{array}$ & $\begin{array}{l}\text { Energy } \\
{[\mathrm{MeV}]}\end{array}$ & $\begin{array}{l}\text { Yield per } \\
\text { decay } \\
{[\%]}\end{array}$ & \begin{tabular}{|l|} 
Air mass \\
energy transfer \\
coeff. \\
{$\left[10^{-3} \mathrm{~m}^{2} \mathrm{~kg}^{-1}\right]$} \\
\end{tabular} & $\begin{array}{l}\text { Air Kerma-rate } \\
\text { const., } \Gamma_{\delta} \\
{\left[\text { aGy m }{ }^{2} \mathrm{~s}^{-1} \mathrm{~Bq}^{-1}\right]}\end{array}$ & $\begin{array}{l}\text { Yield to total } \Gamma_{\delta} \\
{[\%]}\end{array}$ \\
\hline 1 & 2 & 3 & 4 & 5 & 6 \\
\hline \multirow[t]{5}{*}{ I } & 0.1363 & $0.17 \pm 0.02$ & 2.47 & $0.01 \pm 0.001$ & $<0.1$ \\
\hline & 0.2013 & $0.47 \pm 0.04$ & 2.67 & $0.03 \pm 0.003$ & 0.1 \\
\hline & 0.2058 & $3.32 \pm 0.18$ & 2.68 & $0.23 \pm 0.002$ & 0.8 \\
\hline & 0.2832 & $0.27 \pm 0.02$ & 2.85 & $0.03 \pm 0.003$ & 0.1 \\
\hline & \multicolumn{4}{|c|}{$0.30 \pm 0.03$} & $<1.1$ \\
\hline \multirow[t]{4}{*}{ II } & 0.08468 & $28.67 \pm 0.50$ & 2.86 & $3.10 \pm 0.12$ & 10.3 \\
\hline & 0.10011 & $29.65 \pm 0.45$ & 2.88 & $3.35 \pm 0.12$ & 11.2 \\
\hline & 0.11367 & $82.90 \pm 0.4$ & 2.88 & $9.63 \pm 0.28$ & 32.1 \\
\hline & \multicolumn{4}{|c|}{$16.08 \pm 0.44$} & 53.6 \\
\hline \multirow[t]{6}{*}{ III } & 0.3745 & $0.70 \pm 0.03$ & 2.94 & $0.10 \pm 0.01$ & 0.3 \\
\hline & 0.4165 & $0.64 \pm 0.04$ & 2.95 & $0.10 \pm 0.01$ & 0.3 \\
\hline & 0.4681 & $47.94 \pm 0.80$ & 2.97 & $8.50 \pm 0.31$ & 28.4 \\
\hline & 0.4846 & $3.18 \pm 0.15$ & 2.98 & $0.58 \pm 0.04$ & 1.9 \\
\hline & 0.4891 & $0.40 \pm 0.04$ & 2.98 & $0.08 \pm 0.01$ & 0.2 \\
\hline & \multicolumn{4}{|c|}{$9.36 \pm 0.34$} & 31.1 \\
\hline \multirow[t]{4}{*}{ IV } & 0.5886 & $4.47 \pm 0.35$ & 2.97 & $1.00 \pm 0.10$ & 3.3 \\
\hline & 0.6044 & $8.25 \pm 0.45$ & 2.96 & $1.88 \pm 0.14$ & 6.3 \\
\hline & 0.6125 & $5.26 \pm 0.27$ & 2.96 & $1.22 \pm 0.09$ & 4.1 \\
\hline & \multicolumn{4}{|c|}{$4.10 \pm 0.30$} & 13.7 \\
\hline \multirow[t]{4}{*}{$\mathrm{V}$} & 0.785 & $0.05 \pm 0.01$ & 2.88 & $0.01 \pm 0.003$ & $<0.1$ \\
\hline & 0.8845 & $0.29 \pm 0.05$ & 2.88 & $0.09 \pm 0.02$ & 0.3 \\
\hline & 1.062 & $0.06 \pm 0.01$ & 2.76 & $0.02 \pm 0.004$ & 0.1 \\
\hline & \multicolumn{4}{|c|}{$0.12 \pm 0.03$} & 0.5 \\
\hline \multicolumn{5}{|c|}{\begin{tabular}{|l|} 
Total air-kerma rate constant: $30.0 \pm 0.9$ \\
\end{tabular}} & 100.0 \\
\hline
\end{tabular}

Table 3. Data for calculation and calculated Partial, Groups and Total Air kerma rate constant of 192I radionuclide (Ninkovic \& Raicevic, 1993)

As can be seen from Table 3, the entire of photon ray spectrum of ${ }^{192}$ Ir is divided into five characteristic groups of photon lines. The air kerma rate constant was calculated for each discrete photon line with yield per decay event $>0.05 \%$ and starting with energy of 0.1363 $\mathrm{MeV}$ as the lowest energy. That means $\mathrm{X}$-ray were not included. The air kerma rate constant for the groups and for the total were obtained by addition of partial or single photon lines constant. Finally, a value of $(30.0 \pm 0.9)$ aGy m $\mathrm{m}^{-1} \mathrm{~Bq}^{-1}$ for an unshielded ${ }^{192} \mathrm{Ir}$ source has been obtained. That value is in good agreement with a new recalculated value given in Table 1.

Keeping in mind that standard iridium sources are usually packed into $0.15 \mathrm{~mm}$ of platinum, the constant for that type of source was also calculated. For that goal, it was calculated the absorption of iridium photons into $0.15 \mathrm{~mm}$ of platinum and found that in the air kerma rate constant is reduced by $7.33 \%$. After this correction, a value of $(27.8 \pm 0.9)$ 
aGy $\mathrm{m}^{2} \mathrm{~s}^{-1} \mathrm{~Bq}^{-1}$ was obtained for the air kerma rate constant for standard packaged iridium source (Ninkovic \& Raicevic, 1993).

\subsubsection{Results of $\Gamma_{\delta}$ calculation for ${ }^{226} \mathrm{Ra}$ (in equilibrium with its decay product) radionuclide}

\begin{tabular}{|c|c|c|c|c|c|}
\hline $\begin{array}{l}\text { Group of } \\
\text { lines }\end{array}$ & $\begin{array}{l}\text { Energy } \\
{[\mathrm{MeV}]}\end{array}$ & $\begin{array}{l}\text { Yield per decay } \\
{[\%]}\end{array}$ & \begin{tabular}{|l|} 
Air mass \\
energy \\
transfer coeff. \\
{$\left[10^{-3} \mathrm{~m}^{2} \mathrm{~kg}^{-1}\right]$} \\
\end{tabular} & $\begin{array}{l}\text { Air Kerma-rate } \\
\text { const., } \Gamma_{\delta} \\
{\left[\text { aGy m }{ }^{2} \mathrm{~s}^{-1} \mathrm{~Bq}^{-1}\right]}\end{array}$ & $\begin{array}{l}\text { Yield to } \\
\text { total } \Gamma_{\delta} \\
{[\%]}\end{array}$ \\
\hline 1 & 2 & 3 & 4 & 5 & 6 \\
\hline \multirow[t]{14}{*}{$\mathrm{I}$} & 0.1857 & $4.83 \pm 0.26$ & 2.63 & $0.301 \pm 0.020$ & 0.51 \\
\hline & 0.2419 & $8.56 \pm 0.42$ & 2.78 & $0.737 \pm 0.033$ & 1.25 \\
\hline & 0.2588 & $0.49 \pm 0.04$ & 2.28 & $0.046 \pm 0.004$ & 0.08 \\
\hline & 0.2748 & $0.38 \pm 0.15$ & 2.84 & $0.038 \pm 0.013$ & 0.06 \\
\hline & 0.2952 & $19.74 \pm 1.00$ & 2.86 & $2.125 \pm 0.104$ & 3.61 \\
\hline & 0.3520 & $38.27 \pm 2.00$ & 2.92 & $5.015 \pm 0.262$ & 8.51 \\
\hline & 0.3868 & -- & 2.94 & -- & - \\
\hline & 0.3888 & $0.76 \pm 0.14$ & 2.94 & $0.111 \pm 0.020$ & 0.19 \\
\hline & 0.4550 & $0.28 \pm 0.07$ & 2.98 & $0.048 \pm 0.013$ & 0.08 \\
\hline & 0.4621 & $0.16 \pm 0.06$ & 2.98 & $0.028 \pm 0.006$ & 0.06 \\
\hline & 0.4805 & -- & 2.98 & -- & - \\
\hline & 0.4872 & $0.38 \pm 0.05$ & 2.98 & $0.080 \pm 0.007$ & 0.12 \\
\hline & 0.6094 & $46.46 \pm 1.42$ & 2.96 & $10.685 \pm 0.334$ & 18.14 \\
\hline & \multicolumn{4}{|c|}{$19.20 \pm 0.96$} & 32.60 \\
\hline \multirow[t]{14}{*}{ II } & 0.6656 & $1.68 \pm 0.05$ & 2.945 & $0.420 \pm 0.013$ & 0.71 \\
\hline & 0.7031 & $0.58 \pm 0.05$ & 2.935 & $0.135 \pm 0.013$ & 0.26 \\
\hline & 0.7199 & $0.40 \pm 0.05$ & 2.925 & $0.107 \pm 0.013$ & 0.18 \\
\hline & 0.7684 & $4.88 \pm 0.05$ & 2.892 & $1.383 \pm 0.026$ & 2.35 \\
\hline & 0.7860 & $1.10 \pm 0.05$ & 2.89 & $0.319 \pm 0.013$ & 0.54 \\
\hline & 0.8062 & $1.31 \pm 0.04$ & 2.89 & $0.389 \pm 0.013$ & 0.66 \\
\hline & 0.8212 & $0.10 \pm 0.04$ & 2.88 & $0.030 \pm 0.012$ & 0.05 \\
\hline & 0.8392 & $0.52 \pm 0.05$ & 2.875 & $0.160 \pm 0.013$ & 0.27 \\
\hline & 0.9340 & $3.20 \pm 0.15$ & 2.83 & $1.078 \pm 0.033$ & 1.83 \\
\hline & 0.9641 & $0.38 \pm 0,05$ & 2.82 & $0.132 \pm 0.020$ & 0.22 \\
\hline & 0.0520 & $0.35 \pm 0.04$ & 2.78 & $0.130 \pm 0.013$ & 0.22 \\
\hline & 0.1040 & $0.15 \pm 0.04$ & 2.76 & $0.058 \pm 0.012$ & 0.10 \\
\hline & 0.1204 & $16.70 \pm 0.42$ & 2.75 & $6.560 \pm 0.231$ & 11.14 \\
\hline & \multicolumn{4}{|c|}{$10.92 \pm 0.40$} & 18.54 \\
\hline \multirow[t]{5}{*}{ III } & 1.1338 & $0.28 \pm 0.04$ & 2.745 & $0.111 \pm 0.013$ & 0.19 \\
\hline & 1.1553 & $1.58 \pm 0.10$ & 2.735 & $0.636 \pm 0.040$ & 1.08 \\
\hline & 1.2078 & $0.42 \pm 0.05$ & 2.715 & $0.176 \pm 0.020$ & 0.30 \\
\hline & 1.2382 & $6.03 \pm 0.16$ & 2.695 & $2.565 \pm 0.066$ & 4.35 \\
\hline & 1.2811 & $1.52 \pm 0.05$ & 2.675 & $0.664 \pm 0.020$ & 1.13 \\
\hline
\end{tabular}




\begin{tabular}{|c|c|c|c|c|c|}
\hline $\begin{array}{l}\text { Group of } \\
\text { lines }\end{array}$ & $\begin{array}{l}\text { Energy } \\
{[\mathrm{MeV}]}\end{array}$ & $\begin{array}{l}\text { Yield per decay } \\
{[\%]}\end{array}$ & \begin{tabular}{|l|} 
Air mass \\
energy \\
transfer coeff. \\
{$\left[10^{-3} \mathrm{~m}^{2} \mathrm{~kg}^{-1}\right]$} \\
\end{tabular} & $\begin{array}{l}\text { Air Kerma-rate } \\
\text { const., } \Gamma_{\delta} \\
{\left[\text { aGy } \mathrm{m}^{2} \mathrm{~s}^{-1} \mathrm{~Bq}^{-1}\right]}\end{array}$ & $\begin{array}{l}\text { Yield to } \\
\text { total } \Gamma_{\delta} \\
{[\%]}\end{array}$ \\
\hline \multirow[t]{16}{*}{1} & 2 & 3 & 4 & 5 & 6 \\
\hline & 1.3038 & $0.084 \pm 0.035$ & 2.70 & $0.048 \pm 0.024$ & 0.08 \\
\hline & 1.3777 & $4.30 \pm 0.10$ & 2.63 & $1.986 \pm 0.060$ & 3.37 \\
\hline & 1.4016 & $1.31 \pm 0.05$ & 2.62 & $0.613 \pm 0.027$ & 1.04 \\
\hline & 1.4081 & $2.47 \pm 0.05$ & 2.61 & $1.157 \pm 0.033$ & 1.96 \\
\hline & 1.5093 & $2.26 \pm 0.05$ & 2.57 & $1.118 \pm 0.033$ & 1.90 \\
\hline & 1.5387 & -- & 2.56 & -- & - \\
\hline & 1.5433 & $0.29 \pm 0.06$ & 2.555 & $0.146 \pm 0.027$ & 0.25 \\
\hline & 1.5833 & $0.76 \pm 0.05$ & 2.54 & $0.390 \pm 0.027$ & 0.66 \\
\hline & 1.5948 & $0.33 \pm 0.04$ & 2.535 & $0.170 \pm 0.020$ & 0.29 \\
\hline & 1.6055 & $0.33 \pm 0.05$ & 2.53 & $0.171 \pm 0.027$ & 0.29 \\
\hline & 1.6614 & $1.10 \pm 0.04$ & 2.505 & $0.584 \pm 0.020$ & 0.99 \\
\hline & 1.6841 & $0.22 \pm 0.0,04$ & 2.495 & $0118 \pm 0.020$ & 0.20 \\
\hline & 1.7298 & $2.91 \pm 0.11$ & 2.48 & $1.592 \pm 0.060$ & 2.70 \\
\hline & 1.7646 & $16.70 \pm 0.32$ & 2.46 & $9.243 \pm 0.231$ & 15.69 \\
\hline & \multicolumn{4}{|c|}{$21.49 \pm 0.79$} & 36.49 \\
\hline \multirow[t]{11}{*}{ IV } & 1.8386 & $0.34 \pm 0.05$ & 2.43 & $0.194 \pm 0.026$ & 0.33 \\
\hline & 1.8476 & $2.14 \pm 0.09$ & 2.425 & $1.222 \pm 0.043$ & 2.07 \\
\hline & 1.8735 & $0.20 \pm 0.03$ & 2.415 & $0.115 \pm 0.019$ & 0.20 \\
\hline & 1.8967 & $0,06 \pm 0.03$ & 2.405 & $0.035 \pm 0.014$ & 0.06 \\
\hline & 2.011 & $0.05 \pm 0.02$ & 2.36 & $0.030 \pm 0.012$ & 0.05 \\
\hline & 2.017 & - & 2.355 & - & - \\
\hline & 2.119 & $1.24 \pm 0.02$ & 2.325 & $0.779 \pm 0.020$ & 1.32 \\
\hline & 2.2043 & $5.25 \pm 0.03$ & 2.305 & $3.401 \pm 0.119$ & 5.77 \\
\hline & 2.294 & $0.34 \pm 0.01$ & 2.28 & $0.227 \pm 0.007$ & 0.38 \\
\hline & 2.448 & $1.73 \pm 0.03$ & 2.235 & $1.207 \pm 0.033$ & 2.05 \\
\hline & \multicolumn{4}{|c|}{$7.21 \pm 0.30$} & \begin{tabular}{|l|}
12.24 \\
\end{tabular} \\
\hline \multirow[t]{11}{*}{$\mathrm{V}$} & 2.700 & $0.034 \pm 0.005$ & 2.16 & $0.025 \pm 0.026$ & 0.042 \\
\hline & 2.770 & $0.028 \pm 0.004$ & 2.14 & $0.021 \pm 0.043$ & 0.036 \\
\hline & 2.788 & $0.0058 \pm 0.0010$ & 2.135 & $0.001 \pm 0.019$ & 0.02 \\
\hline & 2.885 & $0.0095 \pm 0.0010$ & 2.11 & $0.007 \pm 0.014$ & 0.012 \\
\hline & 2.922 & $0.0162 \pm 0.0030$ & 2.10 & $0.001 \pm 0.0005$ & 0.002 \\
\hline & 2.979 & $0.0162 \pm 0.0010$ & 2.08 & $0.001 \pm 0.0002$ & 0.002 \\
\hline & 3.000 & $0.0089 \pm 0.0010$ & 2.075 & $0.001 \pm 0.0001$ & 0.002 \\
\hline & 3.054 & $0.022 \pm 0.003$ & 2.065 & $0.018 \pm 0.0 .003$ & 0.030 \\
\hline & 3.082 & $0.0047 \pm 0.00005$ & 2.06 & $0.004 \pm 0.001$ & 0.007 \\
\hline & 3.142 & -- & 2.05 & -- & - \\
\hline & \multicolumn{4}{|c|}{$0.08 \pm 0.01$} & 0.14 \\
\hline \multicolumn{5}{|c|}{ Total air-kerma rate constant: $58.9 \pm 2.4$} & 100.0 \\
\hline
\end{tabular}

Table 4. Data for calculation and calculated partial, proup`s and total air kerma rate constant of $226 \mathrm{Ra}$ radionuclide in equilibrium with its decay products (Ninkovic, 1987) 
As it can be seen from this table, the entire of photon ray spectrum of ${ }^{226} \mathrm{Ra}$ (in equilibrium with its decay products) are divided into five characteristic groups of photon lines. The air kerma rate constant was calculated for each discrete photon line with yield per decay event $>0.05 \%$ and starting with energy of $0.1857 \mathrm{MeV}$ as $\delta$ value. That means X-ray were not included. The air kerma rate constant for the groups and for the total were obtained by addition of partial or single photon lines constant. Finally, a value of ( $56.9 \pm 2.4$ ) aGy m² $\mathrm{s}^{-1}$ $\mathrm{Bq}^{-1}$ for an unshielded $226 \mathrm{Ra}$ source has been obtained.

Having seen that standard radium sources are usually packed into $0.5 \mathrm{~mm}$ of platinum, the constant for that type of source was also calculated. For that goal it was used analyses of Shalek and Stoval (Shalek \& Stovall, 1969), which is in good accordance with the earlier estimate of Aglincev et al. (Aglincev et al., 1960), that $0,5 \mathrm{~mm}$ of Pt by absorption of gamma radiation of radium and its decay products, reduce the air kerma rate constant with $9.25 \%$. After this correction, a value of $(53.4 \pm 2.2)$ aGy m$~_{2} \mathrm{~s}^{-1} \mathrm{~Bq}^{-1}$ was obtained for the air kerma rate constant for standard packaged radium sources (Ninkovic, 1987). On the basis of this calculated value and experimentally measured value of Aglincev et al. (Aglincev et al., 1960) it was concluded (Ninkovic, 1987) that the real value of air kerma rate constant of ${ }^{226} \mathrm{Ra}$ in equilibrium with its decay product is smaller by about 1 to $2 \%$, than the value recommended by ICRU (ICRU, Handbook 86, 1963).

\section{Conclussion}

Presented process of recalculation the values for air kerma rate constants, for 35 of the most often used radionuclide in practice, was based on the newest appropriate decay data for every radionuclide and latest numerical data for mass energy-transfer coefficient. That is the reason why, according to the authors opinion, obtained values for $\Gamma_{\delta}$, listed in the table 1 , are the most accurate data that can be found in the literature available at present.

It has to be pointed out that to calculate the absorbed dose to soft tissue the air kerma rate has to be multiplied by the ratio of the mass energy-absorption coefficient of soft tissue to that of air, which can be taken as 1,11 between 2 and $0,1 \mathrm{MeV}$ and drops to 1,04 at $0,02 \mathrm{MeV}$. Also, since the radiation-waiting factor for gamma rays and $X$ rays is 1 , by multiplying air kerma rate constants by a factor 1,11, the soft tissue-equivalent dose constant can be obtained.

\section{References}

Aglincev, K.K, Ostromuhova, G.P. and Holnova, E.A (1960). Izm. Techn. 12, 40

Aird, E.G.A, Williams, S. \& Glover, C. (1984). SI Units and radionuclides: Teaching problems, Radiography, 50, 174-176

Attix, F.H. (1986). Introduction to radiological physics and radiation dosimetry, New York: John Wiley and Sons, Ink

British Committee on Radiation Units and Measurements (1982). Memorandum from British Committee on Radiation Units and Measurements, Br. J. Radiol., 55, 375-377

Fireston,R.B. (1996). Tables of Isotopes, eight edn, New York: John Wiley and Sons

Hubbell, J.H. (1969). Photon Cross Sections, Attenuation Coefficients and Energy Absorption Coefficient from $10 \mathrm{keV}$ to $100 \mathrm{GeV}$, Washington DC: NBS Publication NSRDS-NBS 29 
Hubbell, J.H. \& Seltzer, S.M. (2001). Tables of X-Ray Mass Attenuation Coefficient and Mass Energy Absorption Coefficient from $1 \mathrm{keV}$ to $20 \mathrm{MeV}$ for Elements $Z=1$ to 92 and 48 Additional Substances of Dosimetric Interest, NISTIR 5632, Available at: http://physics.nist. gov./PhysRefData/XrayMassCoef/cover.htmlICRU (1963). Handbook 86, Natl. Bur. Stand. (US)

International Commission on Radiation Protection Units and Measurements. (1962). Radiation Quantities and Units, ICRU Report 10a, Washington DC : ICRU

International Commission on Radiation Protection Units and Measurements (1980). Radiation Quantities and Units, ICRU Report 33, , Washington DC : ICRU

Kereiaces, J.G. \& Rosenstein, M. (1980), Handbook of Radiation Doses in Nuclear Medicine and Diagnostic X-ray Arai, Boca Raton, FL: CRC Press

Nachtigall, D. (1969). Table of Specific Gamma Ray Constants, Muenchen: Verlag Karl Thiemig KG

National Council on Radiation Protection and Measurements (1976). Structural Shielding Design and Evaluation for Medical Use X-rays and Gamma Rays of Energies UP TO 10 $\mathrm{MeV}$. NCRP Report 49, Washington DC: NCRP

Ninkovic, M.M. \& Mladenovic, M. (1970). Specific gamma ray constant and gamma spectrum of ${ }^{192}$ Ir. Atompraxis, 16, 5-8

Ninkovic, .M.M. (1987). The air kerma rate constant of ${ }^{226} \mathrm{Ra}$ in equilibrium with its decay products, Nucl. Instr. Methods, A255, 334-337

Ninkovic, M.M. \& Raicevic J.J. (1992). Air kerma rate constant for the ${ }^{182}$ Ta radionuclide, Trans. Am. Nucl. Soc., V. 65, Suppl. 1, No.9, 70-72

Ninkovic, M.M. \& Raicevic J.J. (1993). The air kerma rate constant of ${ }^{192} I r$, Health Phys. 64(1), 79-81

Ninkovic, M.M, Raicevic, J.J. and Adrovic F. (2005). Air kerma rate constants for gamma emitters used most often in practice, Radiation Protection Dosimetry, 115, 1-4, 247-250

Parker, P.R.. Smith, S.H. \& Taylor, M.D. (1978), Basic Science of Nuclear Medicine, London: Churghill Livingston

Sabol, J. \& Weng, P.S. (1995). Introduction to Radiation Protection Dosimetry, Singapore: World Scientific Publishing Co. Pte. Ltd

Shalek, R.S. \& Stoval M.(1969). in: Radiation Dosimetry Vol.III: Sources, Fields, Measurements and Applications, Attix, F.H., Roesch, W.C. and Tochilin, E., p. 748, Academic Press, New York and London

Stabin, M.G. \& Da Luz L.C.Q.P. (2002). Decay data for internal and external dose assessment, Health Physics, 83(4), 471-475

Ungar, L.M. \& Truby, D.K. (1982). Specific Gamma Ray Dose Constants for Nuclides Important to Dosimetry and radiological assessment, Report No. ORNL/RSIC- 45/R1, Oak Ridge National Laboratory, Oak Ridge TN, USA

Wasserman, H. \& Groenwald, W. (1988). Air kerma rate constants for radionuclides, Eur. J. Nucl. Med., 14, 569-571 


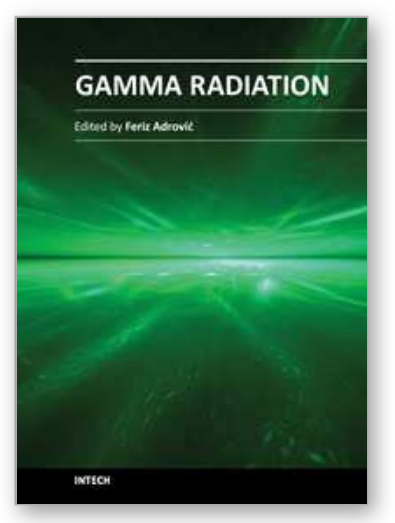

\author{
Gamma Radiation \\ Edited by Prof. Feriz Adrovic
}

ISBN 978-953-51-0316-5

Hard cover, 320 pages

Publisher InTech

Published online 21, March, 2012

Published in print edition March, 2012

This book brings new research insights on the properties and behavior of gamma radiation, studies from a wide range of options of gamma radiation applications in Nuclear Physics, industrial processes, Environmental Science, Radiation Biology, Radiation Chemistry, Agriculture and Forestry, sterilization, food industry, as well as the review of both advantages and problems that are present in these applications. The book is primarily intended for scientific workers who have contacts with gamma radiation, such as staff working in nuclear power plants, manufacturing industries and civil engineers, medical equipment manufacturers, oncologists, radiation therapists, dental professionals, universities and the military, as well as those who intend to enter the world of applications and problems of gamma radiation. Because of the global importance of gamma radiation, the content of this book will be interesting for the wider audience as well.

\title{
How to reference
}

In order to correctly reference this scholarly work, feel free to copy and paste the following:

Marko M. Ninkovic and Feriz Adrovic (2012). Air Kerma Rate Constants for Nuclides Important to Gamma Ray Dosimetry and Practical Application, Gamma Radiation, Prof. Feriz Adrovic (Ed.), ISBN: 978-953-51-0316-5, InTech, Available from: http://www.intechopen.com/books/gamma-radiation/air-kerma-rate-constants-fornuclides-important-to-gamma-ray-dosimetry-and-practical-application-

\section{INTECH}

open science | open minds

\author{
InTech Europe \\ University Campus STeP Ri \\ Slavka Krautzeka 83/A \\ 51000 Rijeka, Croatia \\ Phone: +385 (51) 770447 \\ Fax: +385 (51) 686166 \\ www.intechopen.com
}

\author{
InTech China \\ Unit 405, Office Block, Hotel Equatorial Shanghai \\ No.65, Yan An Road (West), Shanghai, 200040, China \\ 中国上海市延安西路65号上海国际贵都大饭店办公楼 405 单元 \\ Phone: +86-21-62489820 \\ Fax: $+86-21-62489821$
}


(C) 2012 The Author(s). Licensee IntechOpen. This is an open access article distributed under the terms of the Creative Commons Attribution 3.0 License, which permits unrestricted use, distribution, and reproduction in any medium, provided the original work is properly cited. 